\title{
Wireless Sensor Network Energy Efficiency with Fuzzy Improved Heuristic A-Star Method
}

\author{
Sigit Soijoyo \\ Doctoral Program, \\ Department of Computer Science and Electronics \\ Universitas Gadjah Mada, Yogyakarta, Indonesia \\ UniversitasMuhammadiyah, Maluku Utara, Indonesia
}

\author{
Retantyo Wardoyo \\ Department of Computer Science and Electronics \\ UniversitasGadjahMada \\ Yogyakarta,Indonesia
}

\begin{abstract}
Energy is a major factor in designing wireless sensor networks (WSNs). In order to extend the network lifetime, researchers should consider energy consumption in routing protocols of WSNs. Routing will serve to facilitate a number of sensors on the technology of WSNs to identify the optimal path and manage energy consumption saving at the time of transmitting data. Current Wireless Sensor Networks efficiency system uses node selection as the main parameter without applying path finding routing. It will not complete the optimization of energy. This research was designed to address the problem of energy optimization by using fuzzy-improved heuristic A-Star. A new algorithm named improved heuristic AStar was developed from previous A-Star algorithm. The result of fuzzy-improved heuristic A-Star indicated node sensor to sink destination saved 0.3698 joule energy dissipation which resulted in longer lifetime.
\end{abstract}

Keywords-Improved Heuristic A-Star; Fuzzy Logic; Wireless Sensor Network

\section{INTRODUCTION}

A sensor node has limited memory, energy, and resources thus requiring hierarchical settings using clustering so as to lead energy efficiency. Clustering forms an organization of groups called clusters, where each cluster is determined by a node functioning as a head called a cluster head[1]. This cluster head collects data from sensor nodes and sends information to the base station, thus it can be said that the cluster head serves as a bridge between the sensor nodes and the base station and sometimes between cluster heads for the multihop case. Cluster heads are in charge of protecting sensor nodes, adding or removing sensor nodes, scheduling time between sensor nodes, and undertaking data aggregation and message management between nodes and the base station[2].

In this research[3], the approach of fuzzy logic is employed for choosing cluster head Wireless Sensor Networks. It was suggested to improve the lifetime of the network by two times compared to using LEACH and SEP with multi-hop transmission and to modify the technique of choosing cluster head which could extend the lifetime of Wireless Sensor Networks. Based on that research, the present research attempts to compare Fuzzy Sugeno and Fuzzy Mamdani in terms of efficiency in extending the lifetime of Wireless Sensor Networks.
LEACH is a cluster based on classic routing protocol with balanced energy consumption for Wireless Sensor Networks. However, cluster head selection is not based on energy. PEGASIS forms related structure by connecting the node in the farthest edge from the Sink to the node which is closer to the base station. Based on the research, this research attempted to optimize PEGASIS and LEACH as well as fuzzy which only provide the result of sensor node selection. Yet, the algorithm consumes more energy because the farthest node is selected to be the cluster head[6]. The farthest node would be optimized in this research by using A-Star algorithm. However, A-Star had a weakness which is the inaccuracy of heuristic value because it used assumption value.

The process of A-Star algorithm considers the formula

$\mathrm{f}(\mathrm{n})=\mathrm{h}(\mathrm{n})+\mathrm{g}(\mathrm{n})$

In which the algorithm employs assumption value $\mathrm{h}(\mathrm{n})$ as the heuristic value from the sensor node to sink[12]. This research attempted to develop A-Star algorithm from heuristic value assisted by fuzzy algorithm to fill in the weight value of sensor node which had been calculated by fuzzy.

The rest of this paper is organized as follows. Section 2 describes the research framework of Fuzzy approach and Improved Heuristic A-Star. Section 3 presents the related studies on improving network lifetime. Section 4 introduces and discusses the proposed methodology. Section 5 describes result the simulation. Section 6 concludes this paper.

\section{RESEARCH FRAMEWORK}

Clustering is a critical network management operations that are used to reduce network energy consumption and increasing the lifetime of wireless sensor network[4], so it has an important role in wireless sensor network. Previous research [5]-[11] mostly discussed residual energy and distance of neighboring nodes. For an optimum efficiency of energy, not only it needs the result for reference but also the result of evaluation after the cluster head has been selected. In research[6], the result of PEGASIS, LEACH, and Fuzzy Cluster Head Selection consume too much energy because the farthest node is selected as the cluster head. A solution framework which is needed to achieve the optimized value is presented in Fig 1. 


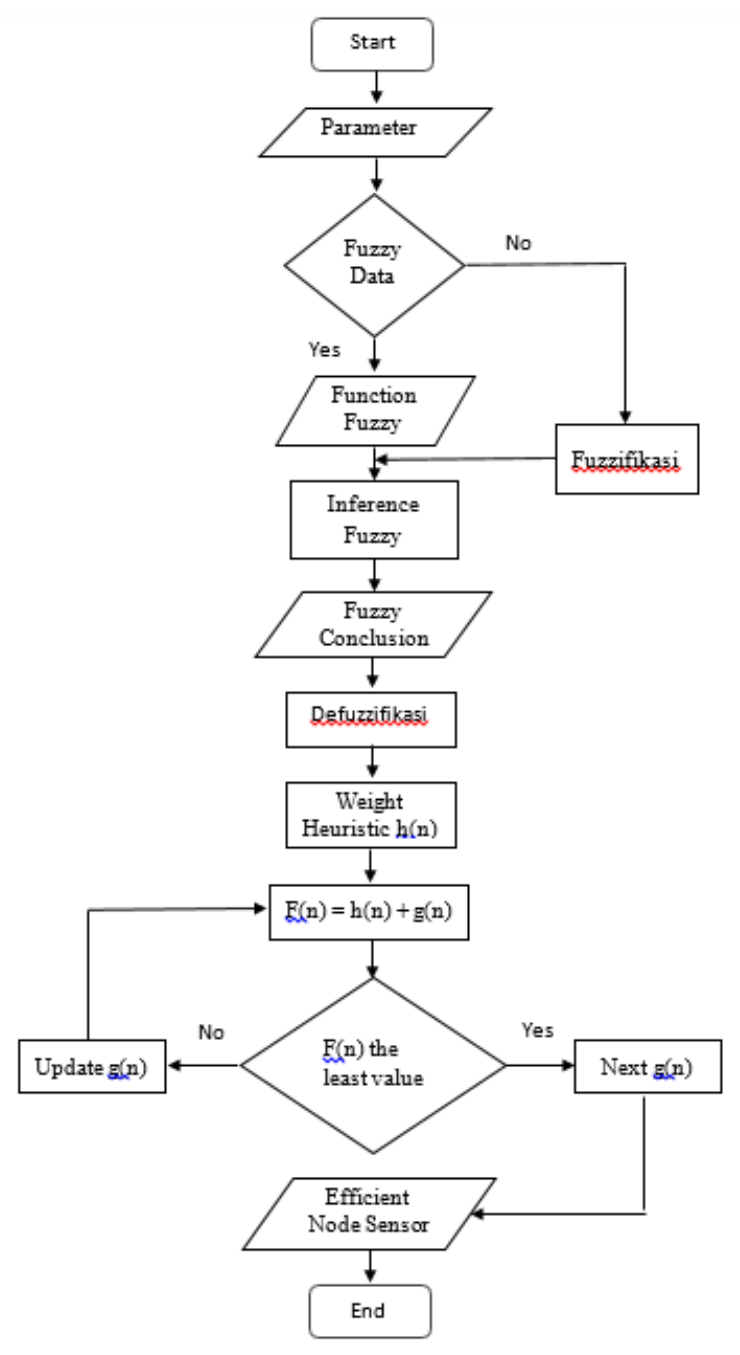

Fig. 1. Solution Framework

\section{RELATED STUDIES}

Gupta et al.[5] in their research using an approach of fuzzy logic to cluster head selection based on three parameters, namely the energy level of each node energy, node concentration and node centrality. In their research, the parameter node centrality played a vital role where the base station selected every single node to be selected as the cluster head by calculating the square of the distance from each node. The lower the centrality value, the lower the amount of energy required by the node to be a cluster head is.

Kim et al.[6] in their research introduced the Cluster Head Election mechanism using Fuzzy Logic (CHEF) to achieve energy efficiency, where a cluster head is selected using two parameters, namely the energy level of each node (energy) and the total distance between each node in the cluster (local distance). The benefits of using CHEF is minimizing the overhead resulting from a centralized process for selection of a cluster head is undertaken locally.

Natajaran and Selvaraj.[6] stated that reselection of cluster head may consume time and energy. Rate parameter of recurrent Communication of Sensor Node (RCSN) is added to solve the problem. RCSN is defined as the number of times a node communicates with its cluster head. In order to emphasize the effect of RCSN to Cluster Head Selection Probability (CHSP), the algorithm of cluster head selection is based on proposed fuzzy. By using RSCN, this algorithm is able to predict energy consumption of node which provides better way for CHSP compared to previous research. This research employs 5 parameters namely Residual Power of Sensor Nodes (RPSN), Degree of Neighbouring Nodes (DNN), Distance between Node and Base Station (DNBS) and Sensor Node Movement (SNM).

Mishra et, al.[7] cluster head selection in wireless sensor network uses fuzzy logic which can solve the problem in CHEF and other cluster head selection protocols. The proposed scheme could help to extend the lifetime of wireless sensor network by selecting the best node for cluster head in order to extend the lifetime of wireless sensor network. This research used residual distance parameter in self-organizing scheme because cluster head has data aggregation responsibility, data processing, data routing, etc. Local distance is the total distance between node $\mathrm{A}$ and its surrounding node. Base station distance is the distance from cluster head to base station and concentration is the direct relationship between cluster head and node member.

Singh et al.[8] unique strategy for cluster head selection in WSN by taking two important parameters namely energy from node and centrality node using fuzzy. This approach had succeeded in significantly extending lifetime compared to LEACH base station approach. According to simulation, this fuzzy scheme extend the lifetime of the network around $70 \%$ compared to LEACH probability approach.

Mhemed et al.[9] introduced a new approach for forming cluster in Wireless Sensor Networks by using Fuzzy Logic to extend the lifetime of the network. The performance is compared with LEACH protocol using FND metric and Paired T-Test in MINITAB. It was found that protocol extended the lifetime from $12 \%$ to $19 \%$. The extension is related with the fact that the protocol used three parameters in the process of forming the cluster while LEACH only used one parameter. The parameters were energy level, distance to base station, and distance to cluster head.

Ando et, al.[10] conclude that cluster head selection becomes difficult when there are many parameters involved in the decision of selecting cluster head. It was suggested to use algorithm of power reduction for wireless sensor based on fuzzy logic and the number of node with simulation system to group algorithm in sensor network. This research used three parameters i.e. Remaining Battery Power of Sensor, Degree of Number of Neighbor Nodes, Distance from Cluster Centroid.

Bidaki and Tabbakh.[11] conclude that fuzzy clustering method changes the selection of cluster head probability in LEACH protocol. The fuzzy system which was used in the inferential machine is fuzzy mamdani which is a simple rule base method. This scheme creates symmetrical cluster and reduce the node of total cluster head distance. It can reduce energy consumption of the sensor and extend the lifetime of Wireless Sensor Networks better than LEACH protocol. The parameters used are neighbour node and remaining energy. The reasons why those parameters are used are: 
- Some nodes which have less neighbour nodes can be selected as the cluster head and the network can be utilized as benefits of data aggregation.

- Each node can independently select itself as cluster head; cluster head can be positioned close to each other.

- Random selection of cluster head does not create symmetrical cluster and it can increase total distance of node cluster head communication. Increasing distance means more energy consumption to transfer data to cluster head which results in shorter lifetime of the network.

Alshawi et, al.[13] concluded that nodes operate by utilizing efficiency of limited energy in battery. The main characteristic of this network is its lifetime that related to the selection of route. Unbalanced energy consumption is an inseparable problem in Wireless Sensor Networks, and proposing new algorithm by using combination of two fuzzy approaches and algorithm A-Star. This new method was able to select optimum routing from source node by supporting the highest residual energy, minimum hop and lowest traffic. The performance of the proposed method is evaluated and compared with other two methods under the same criteria in two different fields of topography. The result of the simulation indicated that the effectiveness of the new method was related to the random extension of the lifetime of the wireless sensor network with node.

Septiana et al.[12] concluded that the process of finding the optimum route in Wireless Sensor Networks routing technique using algorithm A-Star is much dependent on heuristic function. Evaluation function will be more effective if the function has more than one heuristic function. It is because evaluation function conducts more detailed calculation to obtain route cost value by using heuristic A-Star and addition of heuristic value with faster computation time.

Based on research [5]-[13], it could be concluded that majority of the used parameters were energy and distance. However, there is a different research among those research which uses RCSN parameter which is the number of times a node communicates with cluster head. It functions to emphasize the effect of RCSN on Cluster Head Selection Probability algorithm CHSP selection of cluster head based on fuzzy logic. RCSN is claimed to be able to predict better node energy consumption for Cluster Head Selection Probability compared to other parameters. Therefore, in this research the used parameters were RSCN which is the number of times a node communicates with its cluster head, Residual Power of Sensor Nodes (RSPN) which is used in self-organizing scheme because cluster head consumes a lot of energy from sensor node since it has responsibility of data aggregation, data processing, and routing data, Local distance which is the number of distance between node $A$ and node in its surrounding, Base Station Distance which is the distance from cluster head to base station and Remaining Battery Power of Sensor (RPS) which is used to monitor battery left in the sensor. After cluster head is selected, it is optimized by A-Star algorithm. In research [12], the value of $h(n)$ uses estimation, but in in the present research it is in form of real value based on consideration of a number of parameters.
IV. METHODOLOGY

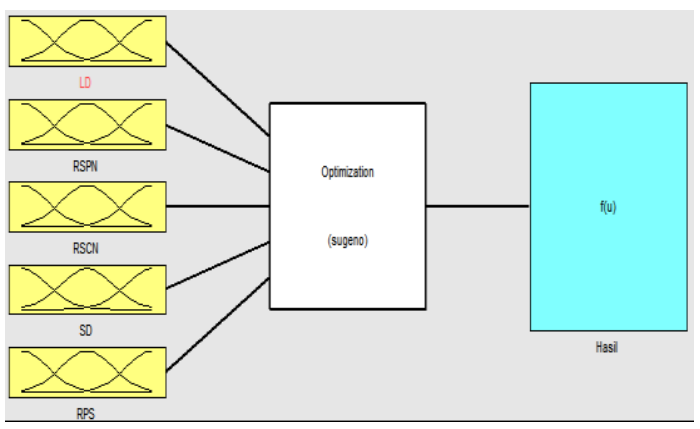

Fig. 2. Variables of the Research

In the present research, fuzzy was used to figure out the heuristic value of $h(n)$ so the value accuration of A-Star was no longer an estimation but a certain value from the used parameters in this research. The variables of the research are

A. Local Distance: the total distance between each node in the cluster.

\section{B. RSPN: Residual Power of Sensor Nodes}

C. RPS: Remaining Battery Power of Sensor

D. Station Distance: Distance to Base Station

E. RCSN: Rate of recurrent Communication of Sensor Node

\section{A. Membership Function of Local Distance}

The compilation of fuzzy of Local Distance membership is presented in Fig 3.

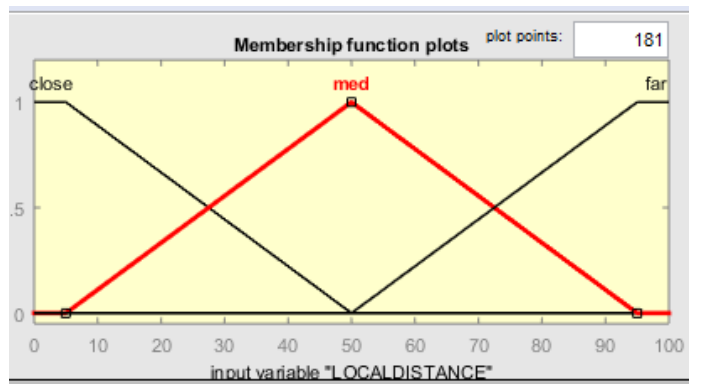

Fig. 3. Local Distance Parameter

$$
\begin{aligned}
& \mu \mathrm{LD}(\text { close })=\left\{\begin{aligned}
0 ; & C \leq 5 \\
\left(\frac{C-5}{50-5}\right) ; & 5 \leq C \leq 50 \\
1 ; & C \geq 50
\end{aligned}\right. \\
& \mu \mathrm{LD}(\text { med })=\left\{\begin{aligned}
0 ; & \mathrm{M} \leq 50 \\
\left(\frac{\mathrm{M}-50}{95-50}\right) ; & 50 \leq \mathrm{M} \leq 95 \\
1 ; & \mathrm{M} \geq 95 \\
0 ; & \mathrm{F} \leq 95
\end{aligned}\right. \\
& \mu \mathrm{LD}(\text { far })=\left\{\begin{aligned}
\left(\frac{\mathrm{F}-95}{100-95}\right) ; & 95 \leq \mathrm{F} \leq 100 \\
1 ; & \mathrm{F} \geq 100
\end{aligned}\right.
\end{aligned}
$$

\section{B. Membership Function of RSPN}

The following Fig 4 presents RSPN parameter 


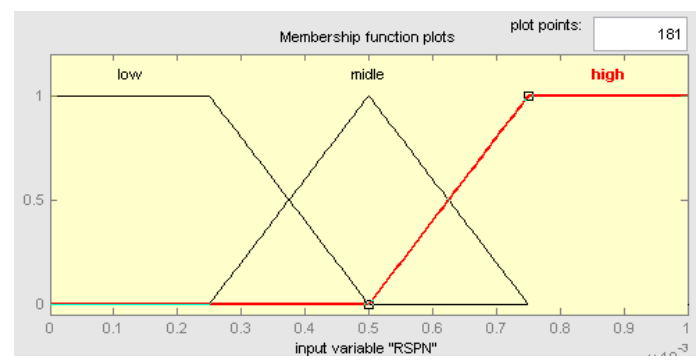

Fig. 4. RSPN Parameter

$\mu$ Low $=\left\{\begin{array}{c}0 ; \mathrm{L} \leq 0 \text { or } \mathrm{L} \geq 0,5 \\ \left(\frac{\mathrm{L}}{0,25}\right) ; 0 \leq \mathrm{L} \leq 0,25 \\ \left(\frac{0,25-\mathrm{L}}{0,5-0,25}\right) ; 0,25 \leq \mathrm{L} \leq 0,5\end{array}\right.$

$\mu$ Midle $= \begin{cases}0 ; M \leq 0,25 \text { or } M \geq 0,75 \\ \left(\frac{M-0,25}{0,5-0,25}\right) ; 0,25 \leq M \leq 0,5 \\ \left(\frac{0,5-M}{0,75-0,5}\right) ; 0,5 \leq M \leq 0,75\end{cases}$

$\mu$ High $=\left\{\begin{array}{c}0 ; \mathrm{H} \leq 0,5 \text { or } \mathrm{H} \geq 1 \\ \left(\frac{\mathrm{H}-0,5}{0,75-0,5}\right) ; \quad 0,5 \leq \mathrm{H} \leq 0,75 \\ \left(\frac{0,75-\mathrm{M}}{1-0,75}\right) ; \quad 0,75 \leq \mathrm{H} \leq 1\end{array}\right.$

C. Membership function of RPS

The compilation of fuzzy of RPS membership is presented in Fig 5.

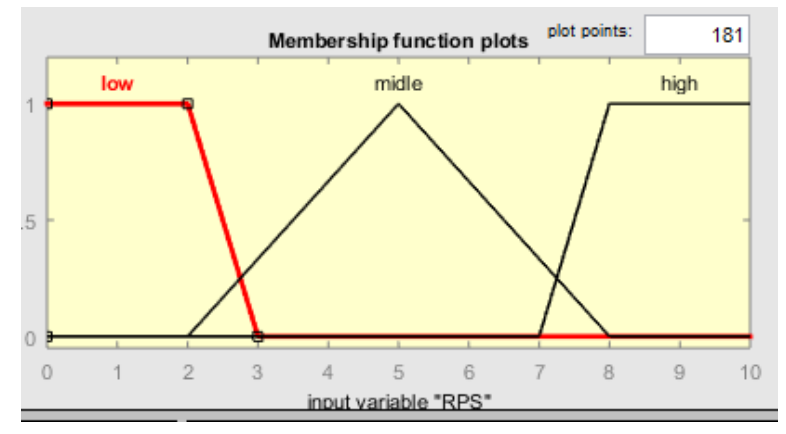

Fig. 5. RPS Speed Parameter

$\mu \operatorname{RPS}($ Low $)=\left\{\begin{aligned} 0 ; & L \leq 2 \\ \left(\frac{\mathrm{L}-2}{3-2}\right) ; & 2 \leq \mathrm{L} \leq 3 \\ 1 ; & \mathrm{L} \geq 3\end{aligned}\right.$

$\mu \operatorname{RPS}($ Midle $)=\left\{\begin{array}{c}0 ; M \leq 5 \\ \left(\frac{S-5}{8-5}\right) ; 5 \leq M \leq 8 \\ 1 ; S \geq 8\end{array}\right.$

$\mu \operatorname{RPS}($ High $)=\left\{\begin{array}{c}0 ; H \leq 8 \\ \left(\frac{H-8}{10-8}\right) ; 8 \leq H \leq 10 \\ 1 ; C \geq 10\end{array}\right.$

\section{Membership Function ofStation Distance}

The compilation of fuzzy of Station Distance membership is presented in Fig 6.

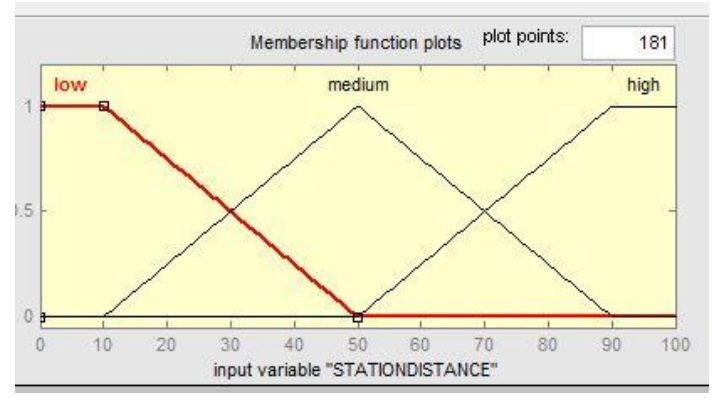

Fig. 6. Station Distance Parameter

$\mu \mathrm{SD}($ Low $)=\left\{\begin{array}{c}0 ; \mathrm{L} \leq 10 \\ \left(\frac{\mathrm{L}-10}{50-10}\right) ; 10 \leq \mathrm{L} \leq 50 \\ 1 ; \mathrm{L} \geq 50\end{array}\right.$

$\mu \mathrm{SD}($ Medium $)=\left\{\begin{array}{c}0 ; M \leq 50 \\ \left(\frac{M-50}{90-50}\right) ; 50 \leq M \leq 90 \\ 1 ; M \geq 90\end{array}\right.$

$\mu \mathrm{SD}($ High $)=\left\{\begin{array}{c}0 ; \mathrm{H} \leq 90 \\ \left(\frac{\mathrm{H}-90}{100-90}\right) ; 90 \leq \mathrm{H} \leq 100 \\ 1 ; \mathrm{H} \geq 100\end{array}\right.$

E. Membership Function of RCSN

The compilation of fuzzy of RCSN is presented in Fig 7.

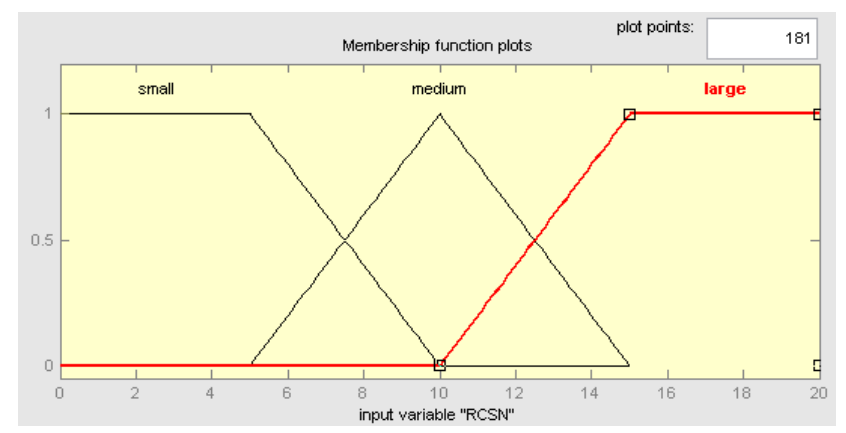

Fig. 7. Parameter RCSN

$$
\begin{aligned}
& \mu \operatorname{RSCN}(\text { Small })=\left\{\begin{array}{l}
0 ; S \leq 0 \text { or } S \geq 10 \\
\left(\frac{S}{5}\right) ; 0 \leq S \leq 5 \\
\left(\frac{5-S}{10-5}\right) ; 5 \leq S \leq 10
\end{array}\right. \\
& \mu \operatorname{RSCN}(\text { Medium })=\left\{\begin{array}{l}
0 ; M \leq 5 \text { or } M \geq 15 \\
\left(\frac{S-5}{10-5}\right) ; 5 \leq M \leq 10 \\
\left(\frac{10-S}{15-10}\right) ; 10 \leq M \leq 15
\end{array}\right.
\end{aligned}
$$




$$
\mu \operatorname{RSCN}(\text { Large })=\left\{\begin{array}{l}
0 ; \mathrm{L} \leq 10 \text { or } \mathrm{L} \geq 20 \\
\left(\frac{\mathrm{L}-10}{15-10}\right) ; 10 \leq \mathrm{L} \leq 15 \\
\left(\frac{15-\mathrm{L}}{20-15}\right) ; 15 \leq \mathrm{L} \leq 20
\end{array}\right.
$$

After all parameters have membership function, a rule is made to evaluate the values of all parameters. It is called rule evaluation[7]. Since there are five parameters and each parameter has three membership attributtes, the rule evaluations are $3^{5}$ which means there are 243 rules.

The next step is making fuzzy final output by using sugeno fuzzy. Output from final output is constant or linear equation

$$
\text { IF }\left(x_{1} \text { is } y_{1}\right) \text { AND ....AND } x_{n} \text { is } y_{n} T_{H E N}
$$

The result of final pocess is obtained by using the following equation

If $\left(A x_{1}\right.$ is $\left.y_{1}\right) \operatorname{AND}\left(B x_{1}\right.$ is $\left.y_{1}\right) \operatorname{AND}\left(C x_{1}\right.$ is $\left.y_{1}\right) \operatorname{AND}\left(D x_{1}\right.$ is $\left.\mathrm{y}_{1}\right)$ AND $\left(\mathrm{E}_{1}\right.$ is $\left.\mathrm{y}_{1}\right) \ldots \ldots$ AND $\left(A \mathrm{x}_{\mathrm{n}}\right.$ is $\left.\mathrm{y}_{\mathrm{n}}\right)$ AND $\left(B \mathrm{x}_{\mathrm{n}}\right.$ is $\left.\mathrm{y}_{\mathrm{n}}\right)$ $\operatorname{AND}\left(\mathrm{C}_{\mathrm{n}}\right.$ is $\left.\mathrm{y}_{\mathrm{n}}\right)$ AND $\left(\mathrm{D} \mathrm{x_{ \textrm {n } }}\right.$ is $\left.\mathrm{y}_{\mathrm{n}}\right)$ AND $\left(\mathrm{Ex}_{\mathrm{n}}\right.$ is $\left.\mathrm{y}_{\mathrm{n}}\right)$ then $\mathrm{Z}=\mathrm{O}$

\begin{tabular}{|c|c|c|c|c|c|c|}
\hline $\begin{array}{c}\text { Rul } \\
\text { es }\end{array}$ & RSPN & $\begin{array}{l}\text { Lokal } \\
\text { distance }\end{array}$ & $\begin{array}{l}\text { Station } \\
\text { distance }\end{array}$ & FPS & RCSN & Output \\
\hline 1 & low & close & high & low & small & low \\
\hline $\begin{array}{l}2 \\
3\end{array}$ & $\begin{array}{l}\text { low } \\
\text { low }\end{array}$ & $\begin{array}{l}\text { close } \\
\text { close }\end{array}$ & $\begin{array}{l}\text { high } \\
\text { high }\end{array}$ & $\begin{array}{l}\text { low } \\
\text { low }\end{array}$ & $\begin{array}{l}\text { medium } \\
\text { large }\end{array}$ & $\begin{array}{l}\text { vlow } \\
\text { vlow }\end{array}$ \\
\hline 20 & low & close & low & low & medium & low \\
\hline 28 & low & medium & high & low & small & vlow \\
\hline 57 & low & far & high & low & large & low \\
\hline 61 & low & far & high & high & small & low \\
\hline 63 & low & far & high & high & large & rlow \\
\hline 82 & midle & close & high & low & small & medium \\
\hline 109 & midle & medium & high & low & small & rhigh \\
\hline 127 & midle & medium & low & low & small & mlow \\
\hline 136 & midle & far & high & low & small & rhigh \\
\hline 190 & high & medium & high & low & small & rlow \\
\hline 217 & high & far & high & low & small & vhigh \\
\hline 218 & high & far & high & low & medium & rhigh \\
\hline 219 & high & far & high & low & large & medium \\
\hline 223 & high & far & high & high & small & rhigh \\
\hline 224 & high & far & high & high & medium & mhigh \\
\hline 225 & high & far & high & high & large & vhigh \\
\hline 226 & high & far & medium & low & small & vhigh \\
\hline 227 & high & far & medium & low & medium & rhigh \\
\hline 228 & high & far & medium & low & large & high \\
\hline 232 & high & far & medium & high & small & medium \\
\hline 233 & high & far & medium & high & medium & medium \\
\hline 234 & high & far & medium & high & large & medium \\
\hline 238 & high & far & low & medium & small & rhigh \\
\hline 239 & high & far & low & medium & medium & high \\
\hline 240 & high & far & low & medium & high & medium \\
\hline 241 & high & far & low & high & small & rhigh \\
\hline 242 & high & far & low & high & medium & medium \\
\hline 243 & high & far & low & high & large & medium \\
\hline
\end{tabular}

TABLE. I. FUZZY RULE BASE

The next step is determine the value of range fuzzy output or fuzzy output chart. Chart of fuzzy output is presented in Fig 8.

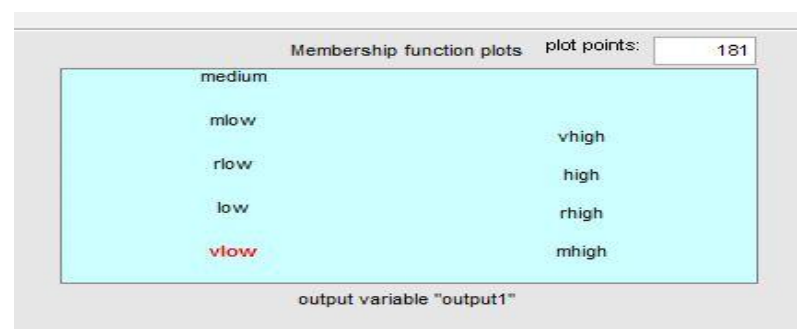

Fig. 8. Range Fuzzy Output
From the Fig 8, the range fuzzy has been determined. The next step is defuzzyfication by using weight average. By using weighted average, each result value of inference to fuzzy rule (W) is multiplied by the value of fuzzy output chart (Z), then the result is summed. The sum is the divided by the sum of final output. Weight average generates real numbers which become the weight of each route Cluster Head Section Wireless Sensor Networks

$$
\text { Final Output }=\frac{\sum_{i=1}^{N}{ }^{w_{i}}{ }_{i}}{w_{i}}
$$

The steps of fuzzy process are complete. The results of fuzzy weight represent each Selected Cluster. If the selected cluster is A and the sink is F, then " $\mathrm{H}$ " represent fuzzy weight to A-B (Hab) and the weight will be considered by A-Star algorithm for efficiency optimization process of Wireless Sensor Networks path. The development of A-Star algorithm is as follows

$$
\begin{aligned}
& f(n)=g(n 1)+g(n 2)-h(\text { weight fuzzy) } \\
& f t(n)=g t(n 1)+g t(n 2)-h t(\text { weight fuzzy) }
\end{aligned}
$$

$\mathrm{f}$ evaluation function is the sum of cost calculation result, where:

- The value of $g(n)$ is the cost paid while moving from source node to neighbor node.

- The value of $h$ (weight fuzzy) is the cost $f$ weight fuzzy needed to move from mediator node to target node.

- $F(t)$ is the total of the cost of $g$ and neighbour heuristic fuzzy if there is branch node which functions to compare the weight of $\mathrm{f}$ to $\mathrm{n}$.

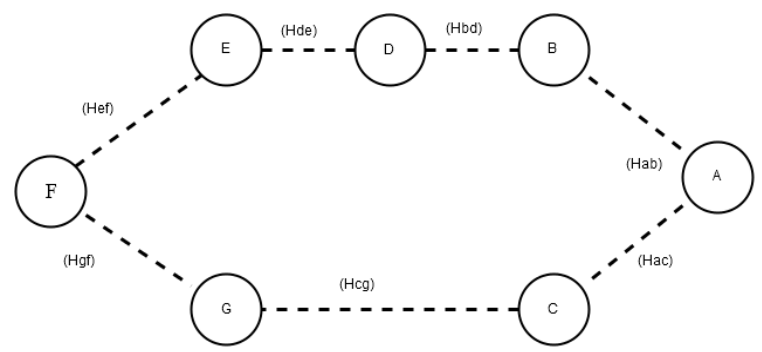

Fig. 9. Cluster Selection Weight with Fuzzy logic

The next step is calculating the formula of enegy disipation[13],

$$
\operatorname{EnT}(k)=\text { Eelec } \bullet+\text { Eamp } \bullet . d^{2}
$$

Transmission and accepting cost is marked by expression Ent $(\mathrm{k})=$ Eelec $\cdot \mathrm{k}+$ Eamp $\bullet \mathrm{k} \cdot \mathrm{d}^{2}$ dan En $\mathrm{R}(\mathrm{k})=$ Eelec $\cdot \mathrm{k}$, where $\mathrm{k}$ is the number of byte per package, $\mathrm{d}$ is the distance from sender node to receiver node, Eelec and Eamp per byte energy dissipation in transmission or accepting circuit and energy needed per byte per square meter as booster to achieve each (SNR) ratio. 


\section{RESULTS AND DisCUSSIONS}

Each node has fuzzy output as weight consideration for cluster head which is presented in Table 2

TABLE. II. TESTING SAMPLE

\begin{tabular}{|l|l|l|l|l|l|l|}
\hline $\begin{array}{c}\text { Node } \\
\text { Sensor }\end{array}$ & RSPN & LD & SD & RPS & RSCN & $\begin{array}{l}\text { Fuzzy } \\
\text { Output }\end{array}$ \\
\hline A & 0.00024 & 12.4 & 18.9 & 1.95 & 2.77 & 0.13 \\
\hline B & 0.00042 & 40.8 & 2.5 & 5.5 & 15.5 & 0.28 \\
\hline C & 0.00083 & 94 & 87.5 & 9.54 & 15.5 & 0.97 \\
\hline D & 0.00012 & 30.9 & 28.9 & 0.67 & 2.06 & 0.20 \\
\hline E & 0.00046 & 45.7 & 48.9 & 4.93 & 14.8 & 0.68 \\
\hline F & 0.00046 & 91.1 & 7.5 & 0.53 & 18.8 & 0.69 \\
\hline G & 0.00090 & 24.5 & 91.1 & 9.75 & 19.1 & 0.86 \\
\hline
\end{tabular}

In Fig 10, candidates of cluster head weight are considered by Heuristic A-Star in order to find the optimum point. Node A-B-D-E-F and A-C-G-F are the probabilities of cluster head selection to be continued to the sink. It uses simulation data where Limit of transmission distance (meters) $30 \mathrm{~m}$, Initial energy of node $0.5 \mathrm{~J}$, Eelec $50 \mathrm{~nJ} / \mathrm{bit}$, Eamp $100 \mathrm{pJ} / \mathrm{bit} / \mathrm{m} 2$, Packet data size 2 bit, Number of transmission packets $2 \times$ 104. The test results are presented in Table 3.

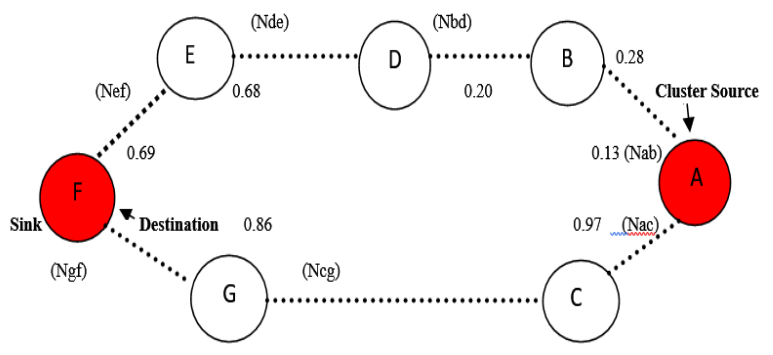

Fig. 10. The Result of Node Sensor Weight

TABLE. III. TEST RESULTNN NVC V

\begin{tabular}{|c|c|c|c|c|}
\hline $\begin{array}{l}\text { Node } \\
\text { Sensor }\end{array}$ & Heuristic & F (n) & $F(t)$ & $\begin{array}{l}\text { Energy } \\
\text { (Joule) }\end{array}$ \\
\hline $\begin{array}{c}\text { A-B-D } \\
\text { (3 Node) }\end{array}$ & 0.69 & 0.08 & 1.83 & 0.0035 \\
\hline $\begin{array}{c}\text { A-C-G } \\
\text { (3 Node) }\end{array}$ & 0.97 & & & 0.2968 \\
\hline $\begin{array}{l}\text { A-B-D-E } \\
\text { (4 Node) }\end{array}$ & 0.69 & 0.27 & 1.83 & 0.0050 \\
\hline $\begin{array}{l}\text { A-C-G-F } \\
\text { (4 Node) }\end{array}$ & 0.97 & & & 0.3928 \\
\hline $\begin{array}{l}\text { A-B-D-E-F } \\
\text { (5 Node) }\end{array}$ & 0.69 & - & - & 0.0230 \\
\hline
\end{tabular}

\section{CONCLUSION AND SUGGESTION}

From this research, it can be concluded that:

- Algorithm hybrid fuzzy-Improved Heuristic A-Star was able to give optimum point which substitutes PEGASIS and LEACH which only considered the selection of cluster head

- Fuzzy with Improved Heuristic A-Star succeed in selecting node sensor which is efficient in energy

- In the experiment, node sensor A-B-D-E consumed energy dissipation 0.0050 joules compared to A-C-G-F 0.3928. Improved Heuristic A-Star algorithm was able to release node based on the most efficient energy dissipation which is A-B-D-E-F 0.0230 joule by saving 0.3698 joule.

- The next research is expected to develop that method by doing experiment in some different topologies for seeing optimum routing result.

\section{REFERENCES}

[1] Jyoti Yadav, Dr. Dubey S.K “Analytical Study of Cluster Head Selection Schemes in Wireless Sensor Networks" International Conference on Signal Propogation and Computer Teknology (ICSPCT), 2014 IEEE,pp.81-85.

[2] Priti Kumari, M.P Singh, Prabhat Kumar "Survey of Clustering Algorithms Using Fuzzy Logic In Wireless Sensor Network" 2013 IEEE

[3] Chourasia, M, K., et al "Energy Efficient Protocol for Mobile Wireless Sensor Networks" 2015 International Conference on Communication, Control and Intelligent Systems(CCIS)978-1-4673-7541-2/15 it 2015 IEEE

[4] Bidaki, M dan Tabbakh S, R,K. 2005 Efficient Fuzzy Logic-Based Clustering Algorithm for Wireless Sensor Networks. IEEE

[5] Vamsi, G, S, M dan Choubey. 2013.A Fuzzy Based Approach of Energy Efficient Hierarchical Clustering Method in Wireless Sensor Networks

[6] Natarajan, H dan Selvaraj, S. 2014. A Fuzzy Based Predictive Cluster Head SelectionScheme for Wireless Sensor Networks. IEEE

[7] Mishra, A,K., Kumar, R., Singh, J. 2015. A novel cluster head selection scheme using fuzzy logic in wireless sensor networks

[8] Singh, A, K., Goutele, S., Verma, S dan Purohit, N. 2012An Energy Efficient Approach for Clustering in WSN using Fuzzy Logic

[9] Mhemed, R., Aslam, N., Phillips, W dan Comeau, F. 2012 An Energy Efficient Fuzzy Logic Cluster Formatin Protocol in Wireless Sensor Networks

[10] Ando, H., Barolli, L., Durresi, A., Xhafa, F dan Koyama, A. 2010. An Intelligent Fuzzy-based Cluster Head Selection System for Wireless Sensor Networks and Its Performance Evaluation

[11] Tabbakh, S, R, K dan Bidaki., M. 2005.Efficient Fuzzy Logic-Based Clustering Algorithm for WirelessSensor Networks

[12] Septiana, R., Soesanti, I dan Setiawan, N, A. 2016. Evaluation Function Effectiveness in Wireless SensorNetwork Routing using A-star Algorithm. IEEE

[13] AlShawi, I, S., Yan, L., Pan, W dan Luo, B. 2012. Lifetime Enhancement in Wireless SensorNetworks Using Fuzzy Approachand A-Star Algorithm. IEEE Sensor Journal, Vol. 12, No. 10, October 2012 